\title{
Heat Shock Protein 90B1 Plays an Oncogenic Role and is a Target of microRNA-223 in Human Osteosarcoma
}

\author{
Guodong Lia,b Ming Caia ${ }^{\mathrm{a}, \mathrm{b}}$ Dong Fu ${ }^{\mathrm{a}}$ Kai Chen ${ }^{\mathrm{a}}$ Mengxiong Sun ${ }^{\mathrm{a}}$ Zhengdong Cai ${ }^{\mathrm{a}}$ \\ Biao Cheng ${ }^{a}$ \\ aDepartment of Orthopaedics, Shanghai Tenth People's Hospital, Tongji University School of Medicine, \\ 301 Yanchang Rd, Shanghai 200072, China; bGuodong Li and Ming Cai contributed equally to this \\ article
}

\section{Key Words}

Osteosarcoma $\cdot \mathrm{HSP} 90 \mathrm{~B} 1 \cdot \mathrm{miR}-223 \cdot \mathrm{PI} 3 \mathrm{~K} \cdot \mathrm{Akt} \cdot \mathrm{mTOR} \cdot$ Apoptosis

\begin{abstract}
Background/Aims: Over the past decade, heat shock protein 90 (Hsp90) has emerged as a potential therapeutic target for cancer. However, the molecular mechanisms of downregulation Hsp90 expression in osteosarcoma are incompletely understood. To develop potential therapy targeting Heat shock protein 90B1 (Hsp90B1), we studied the roles of miR223 in the proliferation and apoptosis of human osteosarcoma. Methods: pcDNA3.1(+)miR-223 plasmid vectors were constructed and transfected into MG63 cells. Co-transfection of miR-223 expression vector with pMIR-Hsp90B1 (The recombined vector of pMIR-GLO ${ }^{\mathrm{TM}}$ luciferase vector containing Hsp90B1-3'UTR) led to the reduced activity of luciferase in a dual-luciferase reporter gene assay, suggesting that Hsp90B1 is a target gene of miR-223. Expression of HSP90B1 was detected by RT-PCR and western blotting analysis. Cell proliferation was determined using the MTT assay. Cell-cycle distribution and apoptosis were examined by flow cytometry. PI3K, p-Akt, Akt, mTOR, Bcl-2 and Bid were also detected by western blotting analysis. After a mouse xenograft model of human MG63 tumors was constructed, tumor growth, microvessel density and proliferation in each group was determined. Results: The pcDNA3.1(+)-miR-223 vector efficiently suppressed the expression of HSP90B1, while silencing miR-223 increased expression of Hsp90B1. Furthermore, overexpression of miR-223 results in significant inhibition of cell growth on culture plates. Moreover, cancer cells showed significant G0/G1 arrest and increased apoptosis due to gene silencing. Protein levels of PI3k, p-Akt, mTOR, and Bcl-2 were decreased, whereas Bid levels were increased. Microvessel density as assessed by CD34 levels and cell growth by PCNA levels decreased according to immunohistochemical analysis. Conclusion: Hsp90B1 is a direct target of miR-223 and miR223 may have a tumor suppressor function in osteosarcoma through the PI3K/Akt/mTOR pathway and could be used in anticancer therapies in osteosarcoma.
\end{abstract}

Copyright $\odot 2012$ S. Karger AG, Basel 


\section{Introduction}

Aside from hematopoietic malignancy, osteosarcoma is the most common primary malignant tumor of bone and can occur in all age groups [1]. Nonetheless, osteosarcoma has a bimodal age distribution, usually occurring in patients between 10 years and 25 years of age $(75 \%)$ and in those over the age of 40 years [2,3]. Effective methods to detect and treat osteosarcoma are lacking: these are extremely important unmet needs that must be addressed.

Heat shock protein 90 (Hsp90) accounts for 1-2\% of protein in a normal, unstressed cell [4]. If cells become stressed, the level of Hsp90 increases. Unsurprisingly, cancer cells typically have elevated levels of Hsp90, accounting for 3-5\% of all the protein in these cells [5]. However, in many human tumor cells, by inhibiting apoptosis and promoting the proliferation of cancer cells, HSP90 facilitates the survival and growth of cancer cells [68]. The specific expression of HSP90 when acting as an anti-apoptotic chaperone protein is commonly higher in cancer cells, including prostate cancer [9], leukemia [10], and lung cancer [11]. HSP90B1 is a member of the HSP90 family of genes. In mammals HSP90B1 protein is also known as glucose-regulated protein 94 (Grp94), which is a homologue of cytosolic HSP90 found exclusively in the endoplasmic reticulum [12]. As glucose deprivation [13], hypoxia [14] and acidosis [15] are hallmarks of solid tumor microenvironments, therefore, overexpression of HSP90B1 in these conditions indicates its possible role as a cancer-related protein.

As a mechanism for the regulation of gene expression, microRNAs (miRNAs) have been paid much attention recently. MiRNAs are a class of small, noncoding RNAs that negatively regulate gene expression by binding to target mRNAs [16]. In osteosaroma, several miRNAs including miR-29b, miR-451, miR-376 and miR-199a-3p are down-regulated [17-19], whereas miR-21, miR181a/b, and miR-150 are up-regulated [18, 20, 21]. TargetScan (http:// www.targetscan.org) was conducted to predict that Hsp90B1 is a target of miR-223 which is abnormally upregulated in several types of cancers, such as ovarian cancer [22], bladder cancer [16] and colorectal cancer [23]. Whereas, miR-223 has been identified as a downregulated miRNA in osteosaroma [18].

Hsp90 has been implicated as an oncogenic agent, but little is known about the exact function of Hsp90B1 as a target of miR-223 in the growth and progression of osteosarcoma. In the present study, we investigated whether Hsp90B1 functions as an oncogene in osteosarcoma by overexpression of miR-223. Our findings confirmed that downregulation of Hsp90B1 inhibits tumor growth in vitro and in vivo. Furthermore, our data showed that Hsp90B1 has an important role in the proliferation and apoptosis of osteosarcoma cells which is mediated by the PI3K/Akt/mTOR pathway.

\section{Materials and Methods}

\section{Plasmid construction}

The eukaryotic expression vector pcDNA3.1(+) (Invitrogen) was used to construct the miR-223 expression plasmid. The miR-223 genomic sequence was amplified by PCR from HEK293 cells (purchased from the Cell Bank of Chinese Academy of Sciences, Shanghai, China). PCR primers for miR-223 were as follows: sense: 5'- TGG ATC CGT GTC ACT CGG GCT TTA CCT G -3' and antisense: 5'- CGA ATT CGT AGA CAC AGC CCA GGG CTG T -3'. The PCR products were digested by EcoR I and BamH I and subcloned into the pcDNA3.1(+) vector. The construct was verified by DNA sequencing.

Culture and transfection of MG63 cells

The human osteosarcoma cell line MG63 was maintained in RPMI 1640 (Pleasanton, CA, USA) supplemented with 10\% fetal bovine serum (FBS; Gibco), 100 units/mL penicillin G, and $100 \mu \mathrm{g} / \mathrm{mL}$ streptomycin (Gibco) at $37^{\circ} \mathrm{C}$ in an atmosphere of $5 \% \mathrm{CO}_{2}$. MG63 cells were seeded in six-well plates with $3 \times 10^{4}$ cells per well 1 day before transfection. When cells were at 70-80\% confluence, miR-223 expression 
vector and pcDNA3.1(+) empty vector or miR-223 inhibitor (Ambion, Austin, TX, USA) were transfected into MG63 cells using Lipofectamine 2000 (Invitrogen, Carlsbad, CA, USA) according to manufacturer's instructions. Cells were collected at different time points according to the purpose of the experiment.

\section{Luciferase reporter assay}

The full length 3'UTR of Hsp90B1 was amplified from a human cDNA library, we used 5'- CAA CGC TTC GGT CAG GGT AT -3' as the upstream primer and 5'- AGG GGG CAT CCA AAA CAA GT -3' as the downstream primer, the amplified product (342 bp) was subcloned into the pMIR-GLO ${ }^{\mathrm{TM}}$ luciferase vector (pMIR, Invitrogen) downstream of the firefly luciferase coding region. The recombined vector was named pMIRHsp90B1. Mutations of miR-223 binding sites were introduced by site-directed mutagenesis from AACUGAC to UUGACUG. The resulting vector was called pMIR-Hsp90B1-Mut.

Luciferase assays were conducted using $1 \times 10^{5}$ HEK293 cells plated on a 96-well plate. Co-transfection was performed using 2 ng pMIR-Hsp90B1, pMIR-Hsp90B1-Mut or pMIR-GLO ${ }^{\text {TM }}$ empty vector and either 80 ng miRNA expression vector or pcDNA3.1(+) empty vector. 24 hours after transfection, the cells were harvested and the protein was extracted for both firefly and renilla luciferase assay using the dual-luciferase glow assay (Promega, Madison, WI). All transfection experiments were conducted in triplicate.

Reverse transcription-polymerase chain reaction (RT-PCR)

Total cellular RNAs were extracted from MG63 cells using Trizol ${ }^{\mathrm{TM}}$ reagent (Invitrogen) according to manufacturer's protocols. The first-strand cDNA was generated by reverse transcription with random hexamer primers and a Superscript RT kit (Invitrogen). Equal amounts of RNAs (3 $\mu \mathrm{g}$ ) were used as templates in each reaction. Quantitative RT-PCR was done with an ABI Prism 7900 Sequence Detection System (PE Applied Biosystems, Foster City, CA, USA). For analysis of Hsp90B1 mRNA levels, we used 5'GAG ATC AAA GAC TAC AGT CCC-3' as the upstream primer and 5'-GTT CGT GCT CAT ACT TGG TC-3' as the downstream primer, as well as 5'-GGC GGC ACC ACC ATG TAC CCT-3' and 5'-AGG GGC CGG ACT CGT CAT ACT3 ' for $\beta$-actin. The PCR reaction for $\beta$-actin and HSP90B1 was determined as 25 cycles, and each cycle was undertaken at $93^{\circ} \mathrm{C}$ for $30 \mathrm{~s}, 52^{\circ} \mathrm{C}$ for $30 \mathrm{~s}$ and $71^{\circ} \mathrm{C}$ for $40 \mathrm{~s}$. The relative expression of HSP90B1 mRNA was calculated using the $2^{-\Delta \Delta C t}$ method, using $\beta$-actin mRNA expression level for normalization. All experiments were repeated at least three times.

\section{Western blotting analysis}

Cells were lysed with RIPA lysis buffer. Proteins were separated by sodium dodecyl sulfatepolyacrylamide gel electrophoresis (SDS-PAGE) and transferred to polyvinylidene difluoride (PVDF) membranes (Millipore, Bedford, MA, USA). Blots were blocked and then probed with antibodies against Hsp90B1 (1:500 dilution; Santa Cruz Biotechnology, Santa Cruz, CA, USA), PI3K(AKT) (1:1,000; Santa Cruz Biotechnology), phospho-AKT (Ser473; 1:400; Santa Cruz Biotechnology), AKT (1:1,000; Santa Cruz Biotechnology), mTOR (1:500; Cell Signaling Technology, Beverly, MA, USA), USA), Bid (1:500; Cell Signaling Technology), Bcl-2 (1:500 dilution; Cell Signaling Technology) and mouse anti- $\beta$-actin, (1:5,000; Santa Cruz Biotechnology). After washing, blots were incubated with horseradish peroxidase-conjugated secondary antibodies and visualized by super ECL detection reagent (Amersham Biosciences, Piscataway, NJ, USA).

\section{Flow cytometry}

Parental MG63 (mock), pcDNA3.1(+) empty vector, and pcDNA3.1(+)-miR-223 groups were harvested by trypsinization and centrifugation, washed twice with cold phosphate-buffered saline (PBS), and fixed with cold $75 \%$ ethanol at $4{ }^{\circ} \mathrm{C}$ overnight. Fixed cells were collected, washed twice with PBS, and suspended in PBS containing $10 \mu \mathrm{g} / \mathrm{mL}$ propidium iodide (Sigma-Aldrich, St Louis, MO, USA) and $100 \mu \mathrm{g} / \mathrm{mL}$ RNase A. They were then incubated at $4^{\circ} \mathrm{C}$ for $\geq 30 \mathrm{~min}$ in the absence of light to eliminate intracellular RNA. Cell-cycle distribution was determined using a flow cytometer.

\section{Cell proliferation assay}

Proliferation of parental cells and stable subline cells was determined using the 3-(4,5-dimethylthiazole2-yl)-2,5-diphenyltetrazolium bromide (MTT) assay. MG63 cells were seeded in 96-well culture plates in culture medium at an optimal density of $7 \times 10^{3}$ cells per well in triplicate wells. Proliferation was analyzed according to the mean value of absorbance. Twenty microliters of $5 \mathrm{mg} / \mathrm{mL}$ MTT substrate was added at 
$37^{\circ} \mathrm{C}$ for $4 \mathrm{~h}$. The colored product was subsequently made soluble in $200 \mu \mathrm{L}$ dimethyl sulfoxide (DMSO). Absorbance was measured at $490 \mathrm{~nm}$ using a microtiter plate reader. Absorbance values were determined at $2,3,4,5$, and 6 days in each group. Each assay was done in triplicate and the experiment was repeated at least three times.

\section{Detection of apoptosis by flow cytometry}

Parental cells and stable subline cells were trypsinized, collected, washed and then stained with annexin V-fluorescein isothiocyanate (FITC; BD Pharmingen, Franklin Lakes, NJ, USA) and propidium iodide (PI; Sigma-Aldrich) for $10 \mathrm{~min}$ at $4^{\circ} \mathrm{C}$ according to a standard protocol from BD Pharmingen. Apoptotic cells were identified by flow cytometric analysis (FACScan; Becton-Dickinson, Franklin Lakes, NJ, USA).

\section{Mouse xenograft model of human MG63 tumors}

Nude mice (4-6 weeks; 18-22 g) were fed on a super-clean biological laminar flow shelf for 1 week. They were then inoculated subcutaneously with $100 \mu \mathrm{L}$ containing $3 \times 10^{6}$ MG63 cells. Tumors were allowed to grow until they reached a diameter of 5-7 mm. Mice were then randomly assigned to three groups of six. Animals received an intratumor injection of $10 \mu \mathrm{g}$ Lipofectamine $2000^{\mathrm{TM}}$-encapsulated miR-223 expression vector, pcDNA3.1 (+) empty vector, or PBS every $3 \mathrm{~d}$. Tumor growth was monitored by measuring the largest (a) and smallest (b) two perpendicular diameters with a caliper. Tumor volume (V) was calculated using the formula:

$$
(V)=a \times b^{2} \times 0.5
$$

\section{Immunohistochemistry}

Mouse xenograft tumor tissues were harvested at the end of treatment and fixed in $10 \%$ neutralbuffered formalin, processed in paraffin, and sectioned at $5 \mu \mathrm{m}$. Tissue sections were then subjected to immunohistochemical staining with antibodies against proliferating cell nuclear antigen (PCNA) and microvessel density (CD34) according to the avidin-biotin complex (ABC) procedure provided by the manufacturer (Vector Laboratories, Burlingame, CA, USA). The areas of positive and negative staining and the number of vessels per unit area $\left(\mathrm{mm}^{2}\right)$ were determined using Image-Pro Plus 5.1 software (Media Cybernetics, Silver Spring, MD, USA).

\section{Statistical analyses}

Results are the mean \pm SD. Data were treated by the Student's $t$-test to determine statistical significance, except for tumor growth data, for which we anticipated a non-normal distribution, and used non-parametric tests (Mann-Whitney test). $\mathrm{P}<0.05$ was considered significant. Statistical analyses were undertaken using SPSS ver12.0 software (SPSS, Chicago, IL, USA).

\section{Results}

MiR-223 interacts with the Hsp90B1 3'UTR and regulates Hsp90B1 protein expression

TargetScan (http://www.targetscan.org) was conducted for miR-223 target prediction. As a result, a binding site of miR-223 was predicted in the 3'UTR of the HSP90B1 mRNA (Fig. 1A). To confirm the specific regulation of Hsp90B1 by miR-223, HEK293 cells were used for the reporter assay. The miR-223 expression vector pcDNA3.1(+)-miR-223 reduced the firefly luciferase activity (Fig. 1B-a). Subsequently, transfections were carried out in HEK293 cells with pMIR-Hsp90B1 and pcDNA3.1(+)-miR-223 or pMIR-Hsp90B1-Mut and pcDNA3.1(+)-miR-223. Mutation of the binding site abolished the ability of miR-223 to inhibit the expression of the luciferase reporter (Fig. 1B-b).

To determine whether miR-223 had a functional role in regulating HSP90B1 gene expression, we created a pcDNA3.1(+)-miR-223 plasmid vector for long-term expression of miR-223 in cells. MG63 cells were transfected with the pcDNA3.1(+)-miR-223. Cells transfected with the empty vector pcDNA3.1(+) were as control. Stable clones were obtained successfully. Overexpression of miR-223 dramatically reduced HSP90B1 expression at both mRNA and protein levels (Fig. 1C and D), while miR-223 silencing by using a miR-223 inhibitor resulted in an increase of Hsp90B1 at protein level (Fig. 1E). 
A

Human HSP90B1 3'UTR

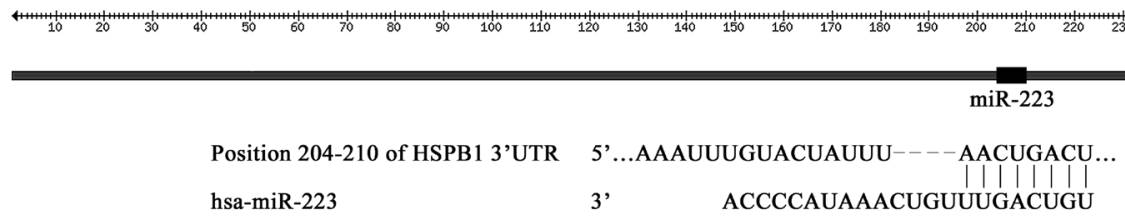

B

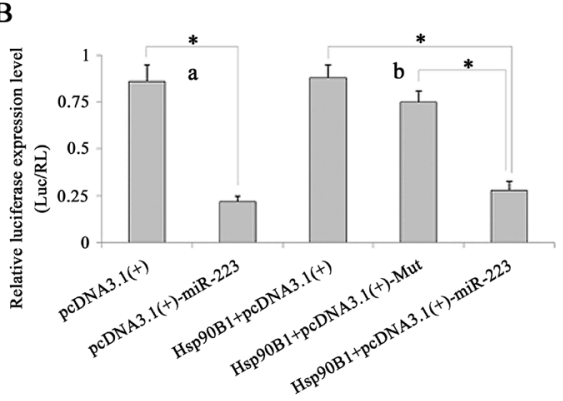

C

D
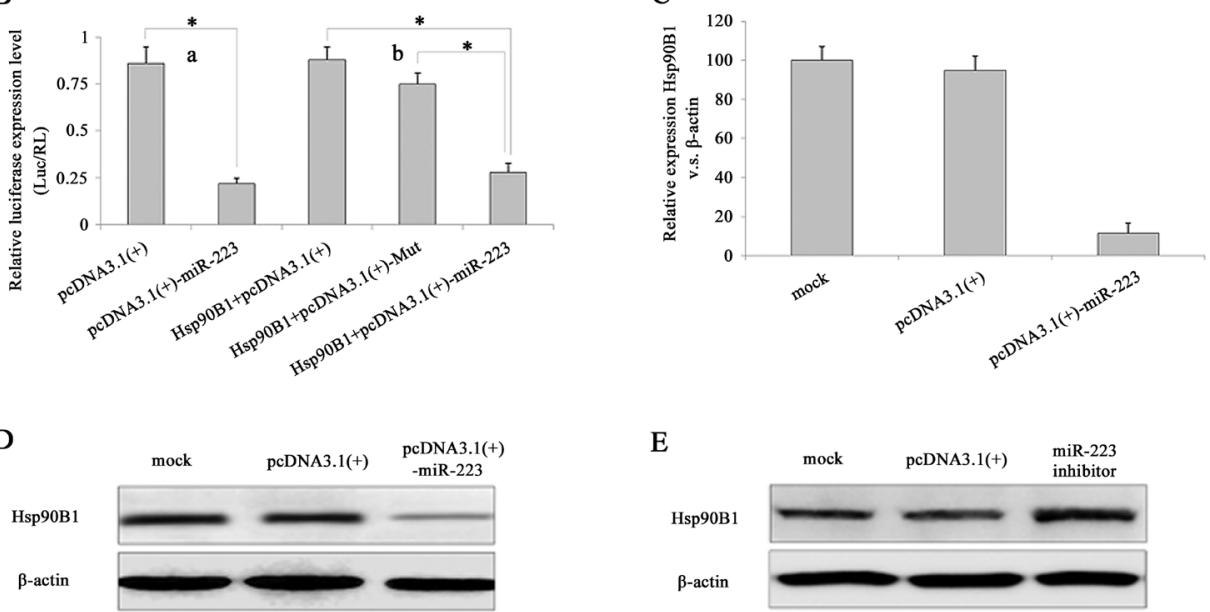

$\mathrm{E}$

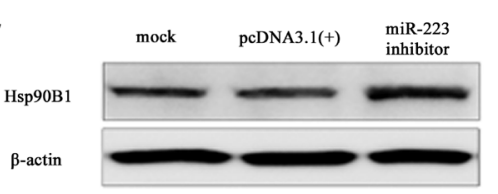

Fig. 1. MiR-223 interacts with the Hsp90B1 3'UTR and regulates the expression of endogenous Hsp90B1. (A) Specific location of the binding site and Hsp90B1 3'UTR. Alignment between the predicted miR-223 target site and miR-223, the conserved 8-bp "seed" sequence for miR-223. mRNA pairing is indicated. (B) The full length $3^{\prime}$ UTR of Hsp90B1 was subcloned into the pMIR-GLO ${ }^{\mathrm{TM}}$ luciferase vector downstream of the firefly luciferase coding region. The miR-223 expression vector pcDNA3.1(+)-miR-223 reduced the activity of firefly luciferase (a). Mutation of the binding site abolished the ability of miR-223 to inhibit the expression of the luciferase reporter (b). (C) Overexpression of miR-223 reduced the mRNA level of Hsp90B1. (D) Overexpression of miR-223 reduced the protein expression of Hsp90B1. (E) miR-223 inhibitor enhanced the protein expression of Hsp90B1. Data are the mean $\pm \mathrm{SD},{ }^{*} \mathrm{P}<0.01$.

\section{Effect of miR-223 on cell proliferation}

Cell proliferation was monitored and analyzed by the MTT assay for 5 days after MG63 cells were stably transfected in mock, pcDNA3.1(+) empty vector and pcDNA3.1(+)miR-223 groups. Relative cell proliferation in mock and pcDNA3.1(+) empty vector group was gradually increased from $24 \mathrm{~h}(0.49 \pm 0.06$ and $0.47 \pm 0.05)$ to $120 \mathrm{~h}(1.71 \pm 0.11$ and $1.67 \pm 0.13)$ in a time-dependent manner, but remained unchanged in cells transfected with pcDNA3.1(+)-miR-223 from $24 \mathrm{~h}(0.49 \pm 0.07)$ to $120 \mathrm{~h}(1.08 \pm 0.11)$. Statistical analyses showed that the plasmid pcDNA3.1(+)-miR-223 could have significant inhibitive effects on the proliferation of MG63 cells compared with the mock control group $(\mathrm{P}<0.001)$ and empty vector control group $(\mathrm{P}<0.001)$. However, the empty vector control group had no significant inhibitive effects on cell proliferation compared with the mock control group $(\mathrm{P}>0.05$, Fig. 2A).

Effect of miR-223 on cell-cycle distribution and apoptosis

To investigate the effects of miR-223 on the cell cycle, G0/G1, G2/M and S phase cells were detected by flow cytometric analysis. MG63 cells were stably transfected with mock, empty vector control and pcDNA3.1(+)-miR-223 groups, and cell proliferation were detected by flow cytometry. In comparison with the mock and vector control groups, the pcDNA3.1(+)-miR-223 group showed cell accumulation in the G0/G1 phase and reduction in the phases of S and G2/M. The total S phase plus G2 phase fraction was less than mock 


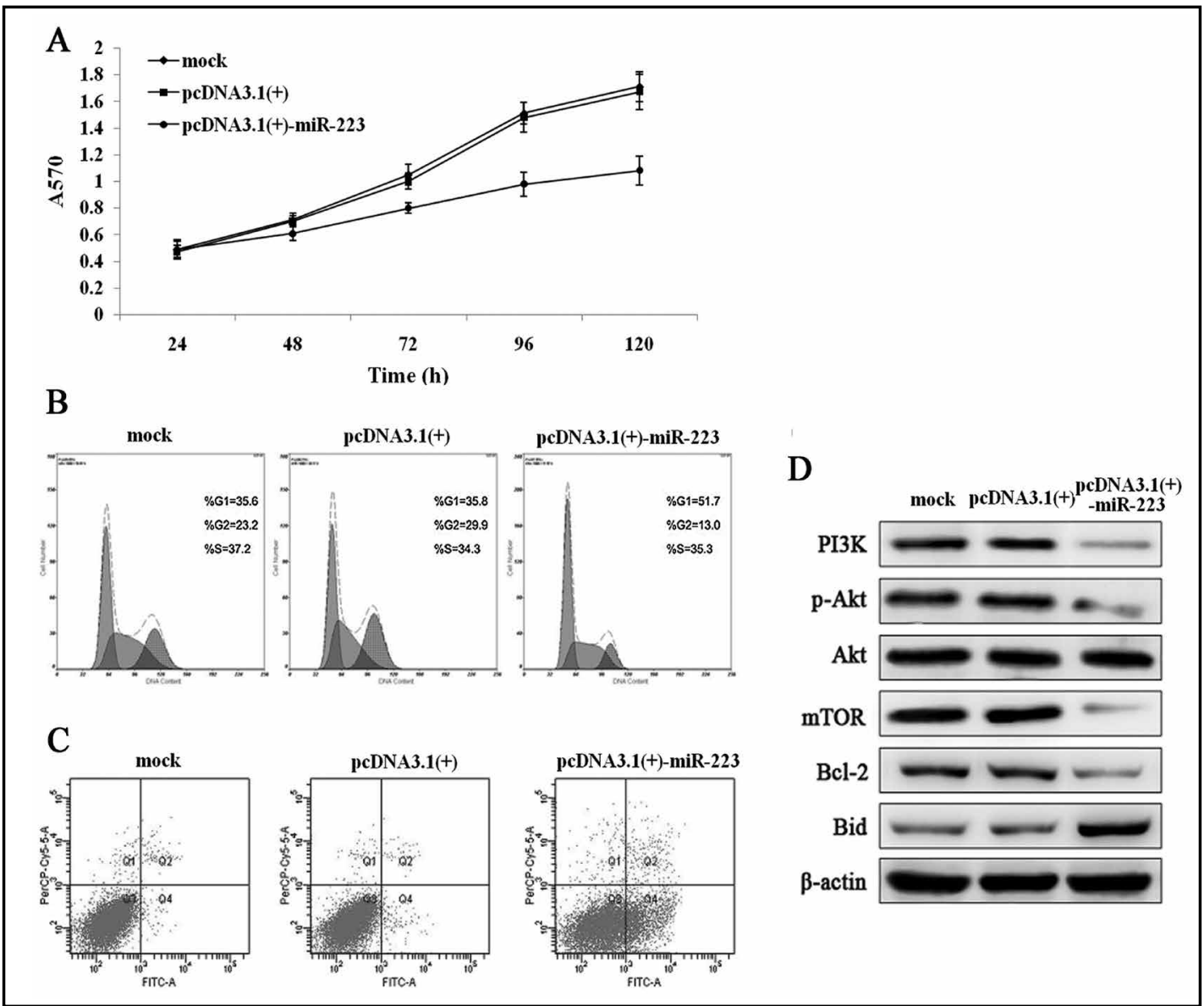

Fig. 2. Effect of miR-223 on cell viability, cell cycle, and apoptosis. (A) Effect of miR-223 on cell viability posttransfection with mock, pcDNA3.1(+) vector and pcDNA3.1(+)-miR-223 vector. (B) Effect of miR-223 on cell cycle. (C) Apoptotic cell death was assessed using the FCM-based annenix V and PI binding assay. (D) The level of PI3K, phospho-AKT, AKT, mTOR, Bcl-2 and Bid post-transfection with mock, pcDNA3.1(+) vector or pcDNA3.1(+)-miR-223 vector in MG63 cells.

$(\mathrm{P}<0.001)$ and empty vector control group $(\mathrm{P}<0.001)$. A significant difference was not found between the mock and empty vector control group (Fig. 2B). Furthermore, to investigate whether the over-expression of miR-223 stable subline increased the apoptosis of MG63 cells, apoptotic cell death was assessed using the FCM-based annenix V and PI binding assay. Flow cytometric analysis also showed that the apoptosis rates of the mock, empty vector control and their stable sublines were $1.17 \% \pm 0.25 \%, 2.35 \% \pm 0.55 \%$, and $21.67 \% \pm 4.5 \%$ respectively. The statistical analysis showed significantly profound rates of apoptotic cell death in pcDNA3.1(+)-miR-223 expressing cells compared with mock $(\mathrm{P}<0.001)$ and empty vector control groups $(\mathrm{P}<0.001)$. However, apoptosis rates in MG63 cells and empty vector control group showed no significant difference (Fig. 2C).

\section{Effect of miR-223 regulates PI3K/Akt/mTOR pathway}

The protein expression levels of PI3K, total-AKT, phospho-AKT (p-AKT), mTOR, Bcl-2 and Bid were examined in mock cells, empty vector control cells, and pcDNA3.1(+)-miR-223 cells. Immunoblot analyses showed that the over-expression of miR-223 led to a significant reduction of PI3K, phospho-AKT, mTOR and Bcl-2, whereas expression levels of the proapoptotic protein Bid were elevated in pcDNA3.1(+)-miR-223 cells (Fig. 2D). 


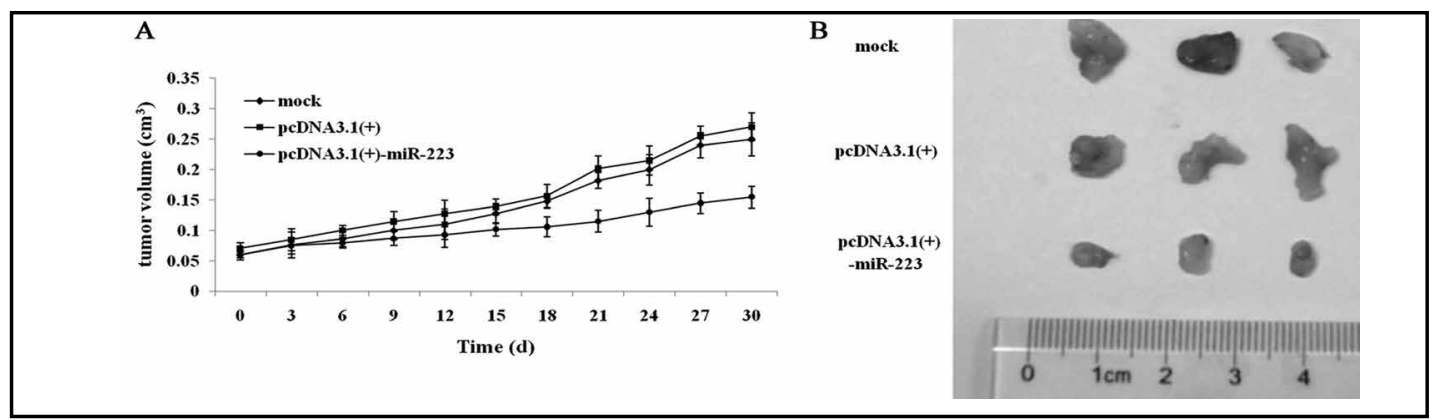

Fig. 3. Effect of miR-223 on the growth of osteosarcoma in vivo. (A) Growth curves of MG63 tumors in nude mice in different treatment groups. (B) Tumor appearances in different treatment groups.

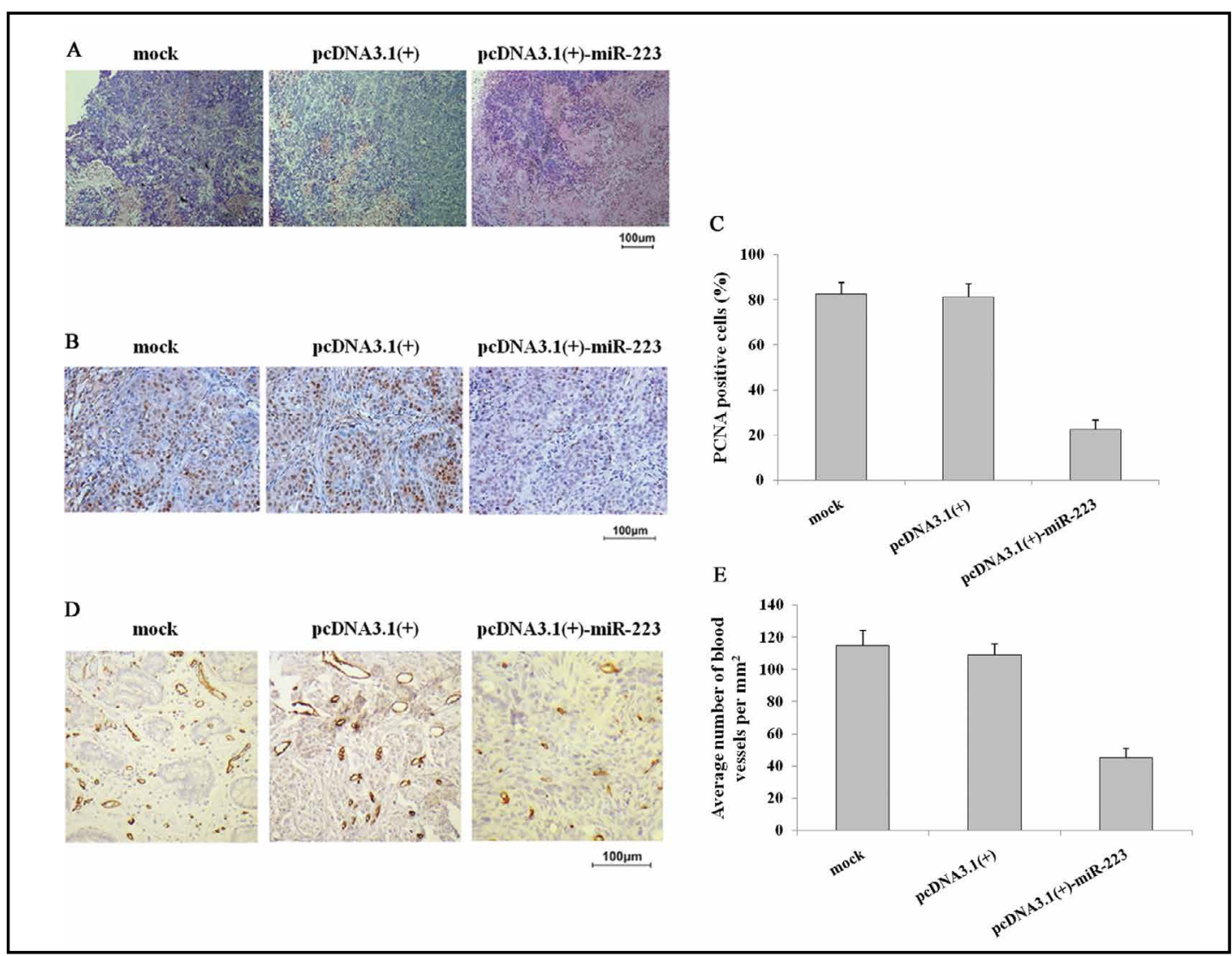

Fig. 4. Paraffin-embedded xenograft tumor sections in different treatment groups were subjected to H\&E staining (original magnification $\times 200$ ). (A) Massive necrotic tissue was found in pcDNA3.1(+)-miR-223 tumors, whereas mock and pcDNA3.1(+) vector control groups showed fine necrosis; (B) Immunosignals for proliferating cell nuclear antigen (PCNA) were dramatically reduced in pcDNA3.1(+)-miR-223-derived tumors compared with the mock and pcDNA3.1(+) vector control groups. (C) The ratio of PCNA-positive cells to total tumor cells was calculated from 5 sections per tumor. Data are the mean \pm SD. (D) CD34 levels were dramatically reduced in pcDNA3.1(+)-miR-223-derived tumors compared with those in mock and pcDNA3.1(+) vector control groups. (E) CD34-positive vessels were enumerated in 5 fields in the central area of each tumor. Data are the mean \pm SD.

\section{Effect of miR-223 on the growth of human osteosarcoma cells in vivo}

To examine the effect of miR-223 on tumor growth in vivo, we established xenografts in nude mice with parental MG63 cells and the subline. Tumor size was monitored every 3 days with a caliper. Tumor growth of the xenografts derived from vector control cells was similar with that in the mock group. However, the tumor volume in the mock and empty vector control groups was markedly increased 12 days after the inoculation of tumor cells 
compared with that in the pcDNA3.1(+)-miR-223 group (Fig. 3A, $\mathrm{P}<0.01)$. Xenografts derived from pcDNA3.1(+)-miR-223 group showed a very slow pattern compared with those in the mock and empty vector control groups $(\mathrm{P}<0.01)$, indicating that over-expression of miR-223 led to growth inhibition of M63 tumors in vivo (Fig. 3A and B). To understand the underlying mechanism, hematoxylin and eosin (H\&E) staining showed that pcDNA3.1(+)-miR-223 tumors were pale, with a massively necrotic center (Fig. 4A). Tumor sections were subjected to immunohistochemical analysis of cellular markers for measurement of cell proliferation and capillary density. Immunosignals for PCNA, a well-defined marker of cell proliferation, were dramatically reduced in pcDNA3.1(+)-miR-223-derived tumors compared with those in the mock and empty vector control groups $(\mathrm{P}<0.01$, Fig. $4 \mathrm{~B}$ and $\mathrm{C})$. CD34 levels were dramatically reduced in pcDNA3.1(+)-miR-223-derived tumors compared with those in the mock and empty vector control groups ( $\mathrm{P}<0.01$, Fig. $4 \mathrm{D}$ and $\mathrm{E})$.

\section{Discussion}

In 1962, Ritossa et al. [24, 25] noticed a different "puffing" pattern of Drosophila chromosomes in response to a sudden increase in incubator temperature, and thereby identified HSPs. Products of HSP genes are, in general, classified according to their molecular weight, for example, HSP110, HSP90, HSP70, HSP60, and a family of small HSPs (HSP27). Among these investigational options, the molecular chaperone HSP90 seems to be one of the most attractive targets for the design and development of drugs [26-28]. Some HSPs in osteosarcoma have been studied. According to Uozaki et al., the overexpressions of HSP27, HSP60, and HSP70 are significantly related to a poor prognosis, and that HSP27 has the strongest negative prognostic value for conventional osteosarcoma. HSP90 is strongly expressed in most cases of conventional osteosarcoma (88.5\%), but not in low-grade central osteosarcoma [29].

Recently, emerging evidence has implicated miRNAs in the proliferation and apoptosis of human cancer cells. MiR-223 was found to be down-regulated in osteosarcoma tissues compared with normal tissues. To verify whether Hsp90B1 expression is regulated by miR223, the pcDNA3.1(+)-miR-223 vector was transfected into MG63 cells. Results from RTPCR and western blotting demonstrated that HSP90B1 mRNA and protein expression were markedly downregulated by overexpression of miR-223, and subsequently resulted in a decrease in cell viability, cell cycle, and increase in apoptosis in vitro compared with the mock and empty vector control groups. These findings suggested that miR-223 targeting Hsp90B1 showed anti-proliferation and pro-apoptotic functions. In addition, MG63 cells transfected with pcDNA3.1(+)-miR-223 vectors grew slowly in vivo in nude mice as compared with the mock and empty vector control groups. H\&E-stained sections indicated that there were several necrotic areas and karyopyknosis was displayed. Immunohistochemical analyses showed that the staining intensities for PCNA and CD34 were lower than those of mock and empty vector control groups.

Phosphatidylinositol 3-kinases (PI3Ks) are divided into four classes: IA, IB, II and III. They are important kinases regulating the survival, proliferation and differentiation of cells [30-32]. Wu et al. [33] found that inhibition of the PI3K/AKT pathway after BMI-1 knockdown played a part in the sensitivity of SAOS-2 cells to cisplatin treatment. Cumulative evidences suggest that abnormal activation of the PI3K/Akt/mTOR signaling pathway occurs frequently in human malignancies [34-36].

Activation of the PI3K/AKT pathway enhances resistance to apoptosis in a wide variety of cancers $[37,38]$. In the present study, inhibition of the PI3K/AKT/mTOR pathway after overexpression of miR-223 was found to play a part in the sensitivity of MG-63 cells. Although the mechanism of apoptosis appears to be related to the PI3K/AKT pathway, how the PI3K/AKT pathway is regulated remains unclear. One possible mechanism is that reduced expression of HSP90B1 after miR-223 overexpression inhibits Akt-Hsp90 binding, and then leads to the dephosphorylation and inactivation of Akt and as a result [39], the PI3K/AKT 
pathway is blocked. In addition, the present study showed a reduced PI3K protein level after miR-223 overexpression, which may be a possible mechanism of how miR-223 inhibits AKT signaling. Third, the ratio of the level of anti-apoptotic to pro-apoptotic proteins is important for the determinination of the extent of apoptosis and survival of cells. We found decreased levels of BCL-2 and Bid accumulation in pcDNA3.1(+)-miR-223 cells, suggesting that miR223 may regulate the ratio of BCL-2 to Bid in MG63 cells by the PI3K/AKT/mTOR pathway.

In summary, the present study demonstrated that miR-223 targeting HSP90B1 could specifically mediate HSP90B1 gene silencing. This effect could inhibit the growth and promote the apoptosis of the osteosarcoma cell line MG63 in vitro and in vivo. A combination of chemotherapy and down-regulation of HSP90B1 by overexpression of miR-223 could be a therapeutic strategy for osteosarcoma.

\section{Disclosure Statement}

All authors are in agreement with the content of this manuscript. The authors declare no conflict of interest.

\section{Acknowledgements}

This study was supported by National Natural Science Foundation of China (NO. 81202116).

\section{References}

1 Enneking WF: Editorial: Osteosarcoma. Clin Orthop Relat Res 1975:2-4.

2 Brooks S, Starkie CM, Clarke NM: Osteosarcoma after the fourth decade. A clinico-pathological review. Arch Orthop Trauma Surg 1985;104:100-105.

-3 Kozakewich H, Perez-Atayde AR, Goorin AM, Wilkinson RH, Gebhardt MC, Vawter GF: Osteosarcoma in young children. Cancer 1991;67:638-642.

4 Welch WJ, Feramisco JR: Purification of the major mammalian heat shock proteins. J Biol Chem 1982;257:1494914959.

5 Crevel G, Bates H, Huikeshoven H, Cotterill S: The drosophila dpit47 protein is a nuclear hsp90 co-chaperone that interacts with DNA polymerase alpha. J Cell Sci 2001;114:2015-2025.

6 Jia W, Yu C, Rahmani M, Krystal G, Sausville EA, Dent P, Grant S: Synergistic antileukemic interactions between 17 aag and ucn-01 involve interruption of raf/mek- and akt-related pathways. Blood 2003;102:1824-1832.

7 Neri LM, Borgatti P, Capitani S, Martelli AM: The nuclear phosphoinositide 3-kinase/akt pathway: A new second messenger system. Biochim Biophys Acta 2002;1584:73-80.

$\rightarrow 8$ Williams CR, Tabios R, Linehan WM, Neckers L: Intratumor injection of the hsp90 inhibitor 17aag decreases tumor growth and induces apoptosis in a prostate cancer xenograft model. J Urol 2007;178:1528-1532.

$>9$ Vanaja DK, Mitchell SH, Toft DO, Young CY: Effect of geldanamycin on androgen receptor function and stability. Cell Stress Chaperones 2002;7:55-64.

10 Flandrin P, Guyotat D, Duval A, Cornillon J, Tavernier E, Nadal N, Campos L: Significance of heat-shock protein (hsp) 90 expression in acute myeloid leukemia cells. Cell Stress Chaperones 2008;13:357-364.

-11 Senju M, Sueoka N, Sato A, Iwanaga K, Sakao Y, Tomimitsu S, Tominaga M, Irie K, Hayashi S, Sueoka E: Hsp90 inhibitors cause $\mathrm{g} 2 / \mathrm{m}$ arrest associated with the reduction of cdc25c and cdc2 in lung cancer cell lines. J Cancer Res Clin Oncol 2006;132:150-158.

12 Graham G, Sharp PJ, Li Q, Wilson PW, Talbot RT, Downing A, Boswell T: Hsp90b1, a thyroid hormone-responsive heat shock protein gene involved in photoperiodic signaling. Brain Res Bull 2009;79:201-207.

13 Li Z: Glucose regulated protein 78: A critical link between tumor microenvironment and cancer hallmarks. Biochim Biophys Acta 2012;1826:13-22. 


\section{Cellular Physiology Cell Physiol Biochem 2012;30:1481-1490 and Biochemistry \\ Li/Cai/Fu/Chen/Sun/Cai/Cheng: Heat Shock Protein 90B1 and microRNA-223 in Osteosarcoma}

14 Krishnamoorthy S, Honn KV: Eicosanoids and other lipid mediators and the tumor hypoxic microenvironment. Cancer Metastasis Rev 2011;30:613-618.

15 Ryder C, McColl K, Zhong F, Distelhorst CW: Acidosis promotes bcl-2 family-mediated evasion of apoptosis: Involvement of acid-sensing g protein-coupled receptor gpr65 signaling to mek/erk. J Biol Chem 2012;287:27863-27875.

16 Gottardo F, Liu CG, Ferracin M, Calin GA, Fassan M, Bassi P, Sevignani C, Byrne D, Negrini M, Pagano F, Gomella LG, Croce CM, Baffa R: Micro-rna profiling in kidney and bladder cancers. Urologic oncology 2007;25:387-392.

17 Kobayashi E, Hornicek FJ, Duan Z: Microrna involvement in osteosarcoma. Sarcoma 2012;2012:359739.

18 Jones KB, Salah Z, Del Mare S, Galasso M, Gaudio E, Nuovo GJ, Lovat F, LeBlanc K, Palatini J, Randall RL, Volinia S, Stein GS, Croce CM, Lian JB, Aqeilan RI: Mirna signatures associate with pathogenesis and progression of osteosarcoma. Cancer Res 2012;72:1865-1877.

19 Lulla RR, Costa FF, Bischof JM, Chou PM, de FBM, Vanin EF, Soares MB: Identification of differentially expressed micrornas in osteosarcoma. Sarcoma 2011;2011:732690.

20 Ziyan W, Shuhua Y, Xiufang W, Xiaoyun L: Microrna-21 is involved in osteosarcoma cell invasion and migration. Med Oncol 2011;28:1469-1474.

21 Lulla RR, Costa FF, Bischof JM, Chou PM, de FBM, Vanin EF, Soares MB: Identification of differentially expressed micrornas in osteosarcoma. Sarcoma 2011;2011:732690.

22 Laios A, O'Toole S, Flavin R, Martin C, Kelly L, Ring M, Finn SP, Barrett C, Loda M, Gleeson N, D'Arcy T, McGuinness E, Sheils O, Sheppard B, J OL: Potential role of mir-9 and mir-223 in recurrent ovarian cancer. Molecular cancer 2008;7:35.

23 Huang Z, Huang D, Ni S, Peng Z, Sheng W, Du X: Plasma micrornas are promising novel biomarkers for early detection of colorectal cancer. International journal of cancer Journal international du cancer 2010;127:118-126. Ritossa F: Discovery of the heat shock response. Cell Stress Chaperones 1996;1:97-98.

Ritossa P: [problems of prophylactic vaccinations of infants]. Riv Ist Sieroter Ital 1962;37:79-108.

Isaacs JS, Xu W, Neckers L: Heat shock protein 90 as a molecular target for cancer therapeutics. Cancer Cell 2003;3:213-217.

27 Mahindra A, Cirstea D, Raje N: Novel therapeutic targets for multiple myeloma. Future Oncol 2010;6:407-418.

28 Takayama S, Reed JC, Homma S: Heat-shock proteins as regulators of apoptosis. Oncogene 2003;22:9041-9047.

29 Moon A, Bacchini P, Bertoni F, Olvi LG, Santini-Araujo E, Kim YW, Park YK: Expression of heat shock proteins in osteosarcomas. Pathology 2010;42:421-425.

-30 Bader AG, Kang S, Zhao L, Vogt PK: Oncogenic pi3k deregulates transcription and translation. Nat Rev Cancer 2005;5:921-929.

31 Engelman JA, Luo J, Cantley LC: The evolution of phosphatidylinositol 3-kinases as regulators of growth and metabolism. Nat Rev Genet 2006;7:606-619.

32 Vivanco I, Sawyers CL: The phosphatidylinositol 3-kinase akt pathway in human cancer. Nat Rev Cancer 2002;2:489-501.

-33 Wu Z, Min L, Chen D, Hao D, Duan Y, Qiu G, Wang Y: Overexpression of bmi-1 promotes cell growth and resistance to cisplatin treatment in osteosarcoma. PLoS One 2011;6:e14648.

-34 Sieghart W, Fuereder T, Schmid K, Cejka D, Werzowa J, Wrba F, Wang X, Gruber D, Rasoul-Rockenschaub S, PeckRadosavljevic M, Wacheck V: Mammalian target of rapamycin pathway activity in hepatocellular carcinomas of patients undergoing liver transplantation. Transplantation 2007;83:425-432.

-35 Villanueva A, Chiang DY, Newell P, Peix J, Thung S, Alsinet C, Tovar V, Roayaie S, Minguez B, Sole M, Battiston C, Van Laarhoven S, Fiel MI, Di Feo A, Hoshida Y, Yea S, Toffanin S, Ramos A, Martignetti JA, Mazzaferro V, Bruix J, Waxman S, Schwartz M, Meyerson M, Friedman SL, Llovet JM: Pivotal role of mtor signaling in hepatocellular carcinoma. Gastroenterology 2008;135:1972-1983, 1983 e1-11.

36 Zhou L, Huang Y, Li J, Wang Z: The mtor pathway is associated with the poor prognosis of human hepatocellular carcinoma. Med Oncol 2010;27:255-261.

37 Brognard J, Clark AS, Ni Y, Dennis PA: Akt/protein kinase b is constitutively active in non-small cell lung cancer cells and promotes cellular survival and resistance to chemotherapy and radiation. Cancer Res 2001;61:3986-3997.

-38 Huang WC, Hung MC: Induction of akt activity by chemotherapy confers acquired resistance. J Formos Med Assoc 2009;108:180-194.

-39 Sato S, Fujita N, Tsuruo T: Modulation of akt kinase activity by binding to hsp90. Proc Natl Acad Sci USA 2000;97:10832-10837. 